INRA Prod. Anim.,

2012,25 (2), 169-180

\title{
Le travail dans les exploitations d'élevage bovin laitier est-il toujours conjugal ?
}

\author{
A. DUFOUR 1, C. GIRAUD 2 \\ 1 ISARA-Lyon, Laboratoire d'études rurales, 23 rue Jean Baldassini, F-69364 Lyon, France \\ 2 Université Paris Descartes, Sorbonne Paris Cité, Cerlis, 45 rue des Saints-Pères, \\ F-75270 Paris, France \\ Courriel : annie.dufour@isara.fr
}

L'agriculture est-elle encore un métier de couple ? Ce slogan est plus ou moins juste selon les orientations technico-économiques des exploitations agricoles. L'élevage laitier reste le secteur le plus «conjugal». En dix ans cependant, l'agriculture s'est globalement «déconjugalisée» avec la volonté des femmes d'acquérir une plus grande indépendance. En nous intéressant à leur parcours biographique et leurs ressources sociales, nous analysons l'expérience et les pratiques très différenciées des femmes qui travaillent en couple sur les exploitations laitières du Ségala.

Le projet de modernisation de l'agriculture conçu dans les années d'aprèsguerre a défendu l'émergence et le développement de l'exploitation familiale à deux Unités de Travail Humain (UTH). Les législateurs ont traduit les aspirations de la fraction moderniste des agriculteurs qui souhaitaient que la famille nucléaire - principalement le couple - joue un rôle clé dans le processus de modernisation des exploitations agricoles. Ce modèle, dans lequel le couple, le travail et l'exploitation sont en étroite interrelation, est tout autant un modèle social qu'un modèle économique : celui d'une agriculture spécialisée intensive (Muller 2009). Ce modèle promeut une autre dimension familiale : la transmission intergénérationnelle de l'exploitation agricole. Ces deux dimensions ne sont pas touchées de la même manière par les évolutions sociologiques récentes des mondes agricoles.

$\mathrm{Au}$ cours des cinquante dernières années, le secteur agricole s'est profondément transformé sur les plans démographique, économique, social et politique. La taille des exploitations s'est accrue, le poids démographique des agriculteurs dans la société française a diminué continûment. La population active agricole, familiale et salariée, atteignait 6,2 millions de personnes en 1955 , soit $31 \%$ de l'emploi total en France. En 2000, cette part est tombée à $4,8 \%$ avec 1,3 million de personnes (Desriers 2007). En 2010, les personnes participant régulièrement au travail des exploitations agricoles ne sont plus que 970000 (Giroux 2011). Les formes d'exercice de l'activité agricole se sont diversifiées. Les exploitations sociétaires $^{1}$ sont en forte croissance depuis 10 ans avec des équipes de travail multiformes (Harff et Lamarche 1998).

Des recherches ont alors montré un éloignement croissant entre la dimension conjugale de la famille et l'exploitation. Le modèle de l'agriculture «métier de couple» a été mis en question (Mundler et Laurent 2003, Bessière et al 2008). L'accroissement du travail à l'extérieur des conjointes de familles agricoles, et la montée, encore modeste, mais significative du salariat agricole dans certaines exploitations ${ }^{2}$ mettent en péril ce modèle. Cette «déconjugalisation» des équipes de travail en agricul- ture est pourtant concomitante de l'amélioration du statut professionnel des conjointes travaillant sur l'exploitation $^{3}$.

La référence idéologique au modèle de l'exploitation familiale est abandonnée en 2005 par le syndicalisme majoritaire au profit de celui de l'entreprise agricole, modèle dans lequel le travail, l'exploitation et la famille sont considérés comme des entités distinctes ${ }^{4}$. Cette évolution se trouve confirmée par la loi d'orientation de 2006 (Rémy 2010). La relation entre le travail et l'exploitation évolue, notamment en raison des transformations de la famille contemporaine. La diffusion des normes d'épanouissement dans le travail et la famille change les rapports à la famille (Singly 2005). La scolarisation massive des filles, qui a connu son plein développement dans les années 1960, permet aux femmes d'être moins dépendantes du mariage et de leur mari (Singly 2007).

Par contraste, la dimension patrimoniale des exploitations agricoles reste toujours très forte comme en témoigne le taux toujours très élevé, proche de

\footnotetext{
1 En 2010, si les petites exploitations agricoles ont presque toutes un statut individuel, 25\% des moyennes et grandes exploitations ont un statut d'EARL contre $14 \%$ dix ans avant (Giroux 2011).

$217 \%$ du travail agricole en 2010 contre $14 \%$ en 2000 seraient assurés par des salariés permanents hors cadre familial (Giroux 2011).

3 Alors que le nombre d'exploitations a baissé de $21 \%$ en dix ans, le nombre de conjoints déclarés «coexploitants» est passé de 26800 en 2000 à 37500 en 2010 (Giroux 2011). Dans les exploitations individuelles, le statut de conjoint collaborateur créé en 2006 a apporté aussi une meilleure reconnaissance du travail des conjoints.

${ }^{4}$ Selon Hervieu et Purseigle (2009), un modèle familial à l'européenne se dessine. Au sein de ce modèle, on a affaire aujourd'hui à une agriculture familiale dont l'activité agricole n'est que l'une des composantes d'un revenu diversifié où les hommes et les femmes de l'exploitation peuvent choisir un métier hors de l'exploitation.
} 


\section{Encadré 1. Méthodologie.}

Notre objet de recherche soulève un double enjeu. II s'agit d'objectiver le travail agricole, de saisir par des indicateurs précis des types d'organisation plus ou moins conjugale mais aussi de mieux comprendre comment ce travail agricole en couple est vécu par les individus eux-mêmes. Le dispositif d'analyse repose sur deux approches complémentaires : une analyse du recensement agricole et une enquête qualitative.

- Le recensement agricole de $2000^{1}$ (RA2000) nous donne des informations sur les équipes de travail en agriculture, la place du salariat et la participation des conjointes qui travaillent dans les exploitations. Ainsi, à partir de ces indicateurs (quotité de travail agricole, importance du salariat), l'analyse statistique permet de caractériser la dimension conjugale du travail dans les exploitations, notamment d'élevage. Le champ retenu pour les statistiques tirées du RA2000 est celui des exploitations individuelles ou sociétaires dont le chef est un homme. En effet, les exploitations où les femmes sont chefs, sont constituées pour moitié d'exploitations dont le chef masculin a pris sa retraite et a concédé ce statut à sa femme. Un quart des exploitations accueille des conjoints masculins avec une activité professionnelle non agricole et un dernier quart connaît des conjoints masculins agriculteurs, ce qui représente $4,6 \%$ des conjoints non coexploitants dans les exploitations professionnelles en 2005 (Rattin 2006). Choisir des exploitations dont le chef est un homme revient donc à supprimer les exploitations de retraite, les exploitations dirigées par des femmes seules et celles, peu nombreuses comparativement à la population totale des exploitations, où les hommes sont des agriculteurs, conjoints non coexploitants. En revanche, la population retenue intègre les exploitations sociétaires où les deux conjoints travaillent et bénéficient d'un statut. Pour le recensement agricole cependant, dans ce cas, c'est le conjoint masculin qui est le plus souvent considéré comme chef d'exploitation ou «premier coexploitant».

- Une enquête qualitative ${ }^{2}$ a été effectuée, courant 2008 , auprès de quatorze femmes qui travaillent dans les exploitations laitières du Ségala (Massif central), notamment dans les départements de l'Aveyron et du Lot. Cette région naturelle se caractérise par son caractère rural, une densité agricole forte et une orientation affirmée vers la production laitière. Les entretiens de type semi-directif visaient à caractériser l'engagement et la place des femmes en élevage laitier. Le choix des femmes interviewées a été effectué en concertation avec des représentants du Comité de développement agricole de Baraqueville (Aveyron), de manière à prendre en compte la diversité des situations des femmes dans les élevages laitiers, des plus engagées dans le travail de l'élevage à celles qui s'en trouvent plus distanciées. La richesse des échanges en début d'entretien a permis l'instauration d'un climat de confiance favorable à la qualité des paroles recueillies. II a été convenu de formuler une question de départ qui invite au récit «Pouvez-vous me parler de vous et de votre activité sur l'exploitation ? Qu'est-ce qui vous a amené à faire ce métier ?» Plusieurs thèmes ont ensuite été explorés : les tâches effectuées, le partage des tâches, la perception des conditions de travail, la représentation du rôle dans l'exploitation, l'insertion sociale et professionnelle. Les entretiens se sont déroulés sans la présence des conjoints. L'âge moyen des femmes interviewées est de 46 ans. Quatre sont titulaires d'un BTS, six du baccalauréat, deux de formations de deux cents heures, deux dont le niveau de formation n'est pas renseigné. Neuf sont d'origine agricole, cinq d'origine non agricole.

1 Le recensement agricole 2010, au moment de la rédaction de cet article, vient de livrer des premiers résultats assez généraux, dont certains viennent d'être rappelés, mais qui sont assez éloignés des vérifications que l'on souhaite réaliser.

2 Les enquêtes ont été effectuées dans le cadre de deux programmes de recherche : le projet TRANS "Transformations de l'élevage et dynamiques des espaces», programme ANR "Agriculture et Développement Durable» et le programme DTL «Dynamique et devenir des Territoires Laitiers» coordonné par la FESIA, et soutenu financièrement par le CNIEL, le Crédit Agricole, GROUPAMA et le SEPROMA.

$85 \%$, des agriculteurs qui sont aujourd'hui fils d'agriculteur. Les relations entre générations se sont cependant transformées en raison de la prégnance des normes d'épanouissement dans le travail qui a conduit les enfants d'agriculteur à ne plus suivre automatiquement la voie tracée par leurs parents, mais à choisir leur profession et à marquer une plus grande autonomie par rapport à la génération antérieure en choisissant parfois de transformer les entrepreneuriale, malgré les efforts déployés pour transformer l'exploitation familiale en entreprise industrielle (Barthez 1982, Dedieu et Servière 1999). Dans ce contexte, cet article interroge la dimension conjugale du travail dans les exploitations agricoles et plus particulièrement dans le secteur professionnellement le plus conjugal qu'est l'élevage laitier : comment s'articulent les rapports de travail et les rapports conjugaux? Quelles représentations les femmes ont-elles de leur travail ? Quel est leur rôle ? C'est à partir d'un croisement de données statistiques et d'enquêtes qualitatives que nous répondrons à ces questions (voir encadré 1).

\section{1 / Une «déconjugalisation» différenciée selon les orien- tations productives}

Comment appréhender concrètement la «déconjugalisation» des équipes de travail agricole? Celle-ci comporte deux dimensions : la première consiste en la participation des conjointes au travail agricole, plus ou moins importante selon l'exploitation; la seconde renvoie à la place que ce travail conjugal occupe par rapport à l'ensemble du travail réalisé sur l'exploitation. La «déconjugalisation» peut ainsi prendre plusieurs formes: la réduction du travail des conjointes, non compensée par celui d'autres acteurs (notamment salariés), la réduction de la part du travail de la conjointe dans l'ensemble du travail réalisé sur l'exploitation.

\section{1 / L'intensité de la participa- tion des conjointes au travail agricole}

La déclaration par la conjointe de sa profession est un premier indicateur de sa participation à l'exploitation. Grâce aux enquêtes du SCEES, nous savons bien que depuis trente ans les femmes ont massivement déserté le terrain de l'exploitation agricole pour embrasser des carrières individuelles dans le monde salarié (Rattin 2002a). Les trois quarts des conjointes les plus jeunes ont une activité professionnelle hors de l'exploitation en 2000 quand elles étaient seulement une sur deux dix ans plus tôt. Cette tendance ne touche cependant pas toutes les exploitations de la même façon. Plus les exploitations agricoles ont une dimension économique importante et plus les équipes de travail sont déconjugalisées 5 : les conjointes choisissent un métier à

\footnotetext{
5 Solange Rattin calcule sur l'ensemble des exploitations agricoles en 2000 que «La proportion de ces ménages pluriactifs (où seul le conjoint est actif non agricole) augmente avec la dimension économique de l'exploitation. Elle est de 5\% dans les exploitations de moins de 8 UDE (1 UDE vaut 1,5 hectare d'équivalent-blé), atteint $21 \%$ quand la dimension est comprise entre 16 et 40 UDE et s'élève à 26\% quand la taille est supérieure à 40 UDE» (2002b, pp. 4-5).
} 
Tableau 1. Importance de deux types de participation féminine au travail selon l'orientation productive des exploitations (OTEX) pour les exploitations dont le chef d'exploitation ou le premier coexploitant est un homme (Source : RA2000).

Toutes les OTEX sont présentées hormis celle intitulée «Exploitations non classées» en raison d'un nombre insuffisant d'exploitations.

\begin{tabular}{|l|c|c|}
\hline \multicolumn{1}{|c|}{ OTEX } & $\begin{array}{c}\text { \% d'exploitations où les conjointes } \\
\text { exercent uniquement une activité } \\
\text { professionnelle à l'extérieur }\end{array}$ & $\begin{array}{c}\text { \% d'exploitations où les conjointes } \\
\text { travaillent au moins à mi-temps } \\
\text { sur l'exploitation }\end{array}$ \\
\hline Bovins lait & 22,7 & 51,9 \\
\hline Bovins lait-viande & 22,5 & 54,9 \\
\hline Poly-élevage/granivores & 26,2 & 46,4 \\
\hline Maraîchage & 20,4 & 36,7 \\
\hline Fleurs/horticulture & 25,2 & 36,1 \\
\hline Poly-élevage/herbivores & 24,5 & 19,3 \\
\hline Polyculture & 24,4 & 20,7 \\
\hline Viticulture d'appellation & 27,3 & 19,6 \\
\hline Autre viticulture & 27,9 & 9,9 \\
\hline Fruits & 29,6 & 14,5 \\
\hline Autres associations & 29,5 & 13,3 \\
\hline Autres herbivores & 35,1 & 13,2 \\
\hline Bovins viande & 34,5 & 19,7 \\
\hline Cultures générales & 34,8 & 25,4 \\
\hline Granivores & 36,5 & 31,1 \\
\hline Grandes cultures et herbivores & 32,2 & 36,4 \\
\hline Céréales/protéagineux & 44,8 & 11,9 \\
\hline Moyenne & 31,9 & 23,7 \\
\hline
\end{tabular}

l'extérieur de l'exploitation tandis que le conjoint masculin assume seul la responsabilité de l'activité agricole.

Mais les femmes peuvent déclarer une profession non-agricole avec un travail plus ou moins important sur l'exploitation. Si l'on combine ces deux informations, on obtient une meilleure mesure de la participation des femmes sur l'exploitation. Le tableau 1 met en regard deux situations opposées, la première est celle des exploitations où les conjointes exercent une activité professionnelle à l'extérieur sans participer au travail de l'exploitation et la seconde est celle des exploitations où les conjointes travaillent entre un mi-temps et un plein temps sans activité extérieure.

Trois secteurs de l'agriculture peuvent être distingués selon le niveau de participation conjugal le plus fréquent :

- Les exploitations en maraîchage, bovins laitiers, bovins laitiers et à viande, polyélevage orientation granivores, fleurs et horticulture favorisent moins que les autres le travail à l'extérieur des conjointes (dans chacune de ces orientations productives, moins d'un quart des exploitations ont une épouse qui n'a pas d'activité sur l'exploitation). Les exploitations en bovins laitiers ou bovins laitiers et à viande ont plus fréquemment que les autres orientations productives un recours intensif (supérieur à un emploi à mi-temps) au travail des conjointes (plus de la moitié des exploitations contre un tiers pour celles de ce premier groupe).

- Les exploitations céréalières, en cultures générales, en grandes cultures et herbivores, en granivores ou en élevage d'autres herbivores ou bovins à viande ont plus souvent que les autres des conjointes qui travaillent exclusivement à l'extérieur (plus d'un tiers des exploitations sont dans ce cas) et participent peu au travail agricole (moins de $40 \%$ des exploitations ont une conjointe y travaillant plus qu'à mi-temps).

- Les exploitations en viticulture d'appellation ou autre, fruits, polyculture ou polyélevage herbivores connaissent moins que les autres des conjointes qui travaillent exclusivement à l'extérieur (environ un quart des exploitations sont dans ce cas) mais elles font également peu appel à elles pour le travail agricole (moins de $20 \%$ des exploita- tions ont des conjointes qui y travaillent plus qu'à mi-temps). Les femmes restent donc plus qu'ailleurs sur l'exploitation avec une participation inférieure ou égale à un emploi à mi-temps.

\section{2 / La place du travail conjugal dans l'ensemble du travail réali- sé sur l'exploitation}

Evaluons maintenant la participation des conjointes en la rapportant au travail agricole total sur l'exploitation et notamment au travail salarié qui peut s'y substituer. Nous utiliserons ici le nombre d'Unités de Travail Annuel (UTA) moyen. Il est en effet possible de calculer le nombre d'UTA moyen du chef d'exploitation, de sa conjointe, des salariés temporaires ou permanents, selon les systèmes de production (OTEX). Nous proposons de comparer pour chaque orientation le ratio entre le nombre moyen d'UTA des conjointes et le nombre moyen d'UTA totales. Le même ratio a été calculé avec le nombre d'UTA salariées (voir le tableau 2) ${ }^{6}$. La prise en compte de cette deuxième

\footnotetext{
${ }^{6}$ Les UTA des coexploitants éventuels ou de la main-d'œuvre familiale sans statut (hors conjoint) prises en compte dans le nombre total d'UTA ne sont pas reproduits dans le tableau 2 .
} 
Tableau 2. Nombre moyen d'Unités de Travail Annuel (UTA, en millièmes) assurées par les conjointes et les salariés selon l'orientation productive des exploitations (OTEX) pour les exploitations dont le chef d'exploitation ou le premier coexploitant est un homme (Source : RA2000).

\begin{tabular}{|l|c|c|c|c|c|}
\hline OTEX & $\begin{array}{c}\text { UTA assurées } \\
\text { par le chef } \\
\text { d'exploitation }\end{array}$ & $\begin{array}{c}\text { UTA assurées } \\
\text { par la } \\
\text { conjointe }\end{array}$ & $\begin{array}{c}\text { UTA assurées } \\
\text { par des } \\
\text { salariés }\end{array}$ & $\begin{array}{c}\text { \% des UTA de } \\
\text { la conjointe } \\
\text { dans les UTA } \\
\text { totales }\end{array}$ & $\begin{array}{c}\text { \%ans les UTA } \\
\text { salariées } \\
\text { totales }\end{array}$ \\
\hline Bovins lait & 966 & 540 & 92 & 28,4 & 4,8 \\
\hline Bovins lait-viande & 969 & 562 & 98 & 27,4 & 4,8 \\
\hline Poly-élevage/granivores & 878 & 488 & 213 & 24,8 & 10,8 \\
\hline Poly-élevage/herbivores & 550 & 240 & 77 & 22,2 & 7,1 \\
\hline Grandes cultures et herbivores & 902 & 398 & 177 & 21,3 & 9,5 \\
\hline Autres herbivores & 465 & 168 & 73 & 21,1 & 9,2 \\
\hline Bovins viande & 729 & 243 & 73 & 20,3 & 6,1 \\
\hline Autres associations & 467 & 168 & 107 & 19,7 & 12,6 \\
\hline Céréales/proteagineux & 715 & 161 & 163 & 13,6 & 13,8 \\
\hline Polyculture & 619 & 254 & 416 & 17,3 & 28,4 \\
\hline Granivores & 907 & 356 & 607 & 17,0 & 28,9 \\
\hline $\begin{array}{l}\text { Cultures générales (betteraves, } \\
\text { pommes de terre...) }\end{array}$ & 872 & 303 & 616 & 14,9 & 30,3 \\
\hline Autre viticulture & 520 & 133 & 317 & 12,7 & 30,3 \\
\hline Maraîchage & 897 & 428 & 1560 & 13,9 & 50,5 \\
\hline Viticulture de qualite & 665 & 269 & 859 & 13,7 & 43,6 \\
\hline Fleurs/horticulture & 915 & 449 & 2091 & 11,9 & 55,7 \\
\hline Fruits & 575 & 202 & 1092 & 10,1 & 54,7 \\
\hline
\end{tabular}

dimension permet d'avoir une vue complémentaire sur le caractère plus ou moins conjugal des équipes de travail, c'est-à-dire sur l'importance du travail conjugal dans l'ensemble du travail de l'exploitation et ce, quelle que soit la quantité globale de travail propre à chaque orientation.

Trois secteurs se distinguent à nouveau :

- Une agriculture caractérisée par une forte mobilisation des conjointes (de 20 à $30 \%$ des UTA sont réalisées par les conjointes) et un recours faible au salariat (moins de 15\% des UTA). Elle regroupe les orientations bovins lait, lait-viande et viande, poly-élevage orientation granivores ou herbivores, autres herbivores, grandes cultures et herbivores et autres associations. Le recours aux conjointes renvoie aux contraintes spécifiques de ces secteurs d'élevage souvent intensif (un fort travail d'astreinte qui peut être difficile à déléguer à des salariés sans des frais conséquents) ainsi qu'à une séparation assez nette des rôles féminins et masculins, les conjointes s'occupant souvent de la traite, de la comptabilité, mais nous en reparlerons dans la seconde partie.
- Une agriculture caractérisée par une participation moyenne des conjointes (de 10 à $20 \%$ des UTA de l'exploitation) et une utilisation modérée de salariés (qui va de un quart à $30 \%$ des UTA). Il s'agit des orientations cultures générales, granivores, polyculture, autre viticulture, etc. Les exploitations céréalières peuvent aussi se rattacher à ce secteur même si l'appel au salariat $y$ est singulièrement plus faible. Ce secteur paraît pouvoir se passer plus facilement qu'ailleurs du travail de la conjointe comme d'une aide extérieure en raison de la mécanisation et fonctionner avec le seul chef d'exploitation (dont le temps en termes d'UTA est assez faible).

- Une agriculture caractérisée par un travail des conjointes qui représente une part réduite dans le travail d'ensemble (moins de $15 \%$ des UTA) et un recours très important au salariat (les salariés permanents ou saisonniers réalisent plus de $50 \%$ des UTA). Elle concerne les secteurs spécialisés de la vigne, du maraîchage, des fruits, des fleurs et de 1'horticulture. La place particulière du maraîchage dans ce secteur s'explique par le fait que la quotité de travail très importante des femmes comparativement à celle, plus faible, d'autres orientations de ce secteur comme la viticulture devient finalement modeste quand on la rapporte à l'ensemble des heures de travail sur les exploitations maraîchères qui font largement appel aux salariés, permanents ou saisonniers. Les femmes y sont souvent responsables des ateliers de tri, de calibrage et de conditionnement des produits et peuvent gérer une main-d'œuvre salariée nombreuse, souvent féminine. Ce troisième secteur est donc caractérisé par des équipes qui mêlent en des proportions diverses travail conjugal et travail salarié notamment saisonnier, nécessaire pour certaines productions très demandeuses en main-d'œuvre. Depuis des décennies, des salariés sont associés aux travailleurs familiaux.

\section{3 / Différentes configurations des équipes de travail}

Ces deux dimensions (intensité de la participation de la conjointe à l'exploitation et importance de sa contribution à l'équipe de travail) permettent de mieux apprécier le caractère plus ou moins 
Tableau 3. Répartition des types d'équipe de travail (\%) selon l'orientation productive des exploitations (OTEX), pour les exploitations dont le chef d'exploitation ou le premier coexploitant est un homme (Source : RA2000).

\begin{tabular}{|l|c|c|c|c|}
\hline OTEX & Equipe conjugale & $\begin{array}{c}\text { Equipe conjugale } \\
\text { et salariée }\end{array}$ & $\begin{array}{c}\text { Equipe salariée } \\
\text { non conjugale }\end{array}$ & $\begin{array}{c}\text { Equipe non conjugale } \\
\text { non salariée }\end{array}$ \\
\hline Bovins lait-viande & 54,8 & 17,3 & 8,3 & 19,6 \\
\hline Bovins lait & 54,9 & 16,3 & 8,0 & 20,8 \\
\hline Grandes cultures et herbivores & 40,1 & 18,9 & 12,1 & 28,9 \\
\hline Poly-élevage/granivores & 39,7 & 25,1 & 10,4 & 24,8 \\
\hline Bovins viande & 38,5 & 9,5 & 7,5 & 44,5 \\
\hline Poly-élevage/herbivores & 35,4 & 10,2 & 6,2 & 48,2 \\
\hline Autres herbivores & 28,7 & 6,6 & 5,1 & 59,6 \\
\hline Céréales/protéagineux & 24,2 & 12,7 & 12,7 & 50,4 \\
\hline Autres associations & 23,0 & 11,2 & 7,5 & 58,3 \\
\hline Polyculture & 25,6 & 21,2 & 17,3 & 35,9 \\
\hline Granivores & 24,7 & 28,6 & 21,5 & 25,2 \\
\hline $\begin{array}{l}\text { Cultures génerales (betteraves, } \\
\text { pommes de terre...) }\end{array}$ & 25,5 & 26,8 & 23,1 & 24,6 \\
\hline Fruits & 21,2 & 24,9 & 23,2 & 30,7 \\
\hline Autre viticulture & 16,5 & 18,5 & 25,8 & 39,2 \\
\hline Maraîchage & 28,0 & 34,9 & 20,8 & 16,3 \\
\hline Fleurs/horticulture & 24,2 & 38,5 & 22,7 & 14,6 \\
\hline Viticulture de qualité & 9,5 & 42,2 & 32,2 & 16,1 \\
\hline Moyenne & 30,3 & 18,1 & 14,1 & 37,5 \\
\hline
\end{tabular}

conjugal des exploitations agricoles? Quatre configurations vont être distinguées :

- Une agriculture où les équipes de travail se limitent à la famille et surtout au couple exploitant et font appel au salariat pour moins de $20 \%$ des UTA («équipes conjugales» présentes dans $28,7 \%$ des exploitations).

- Une agriculture où les équipes mêlent travail familial, conjugal, et travail salarié qui représente plus de $20 \%$ des UTA («équipes conjugales et salariées», soit 15,8\% des exploitations).

- Une agriculture où les équipes de travail font appel largement au salariat (plus de 20\% des UTA) mais pas à la conjointe («équipes salariées non conjugales», soit $16,3 \%$ des exploitations).

- Une agriculture où les équipes de travail ne font pas appel au conjoint, et font appel au salariat pour moins de $20 \%$ des UTA («équipe non conjugale non salariée», limitée au chef d'exploitation, soit 39,2\% des exploitations).
Le tableau 3 présente la façon dont ces différentes équipes de travail sont distribuées en fonction des orientations productives.

Les éleveurs (surtout dans les orientations bovins lait et bovins lait-viande) sont beaucoup plus enclins que les autres agriculteurs à travailler en équipe conjugale. Les autres orientations d'élevage s'appuient aussi plus souvent que la moyenne (mais moins qu'en bovins laitiers et en bovins laitiers et à viande) sur une équipe conjugale. Mais il faut distinguer les orientations comme bovins viande, autres herbivores, polyélevage herbivores qui vont s'appuyer plus souvent que les autres sur des équipes non-conjugales, non-salariées, souvent limitées au seul chef d'exploitation et avoir moins recours aux équipes salariées (à la différence des orientations poly-élevage granivores ou à l'orientation grande culture et herbivores).

La prise en compte de la quotité de travail des conjointes qui travaillent plus d'un mi-temps permet d'affermir et de préciser cette distinction interne au groupe des orientations productives à dominante conjugale : les orientations qui ont le plus tendance à se trouver dans une configuration conjugale où les conjointes travaillent plus d'un mitemps sont les bovins lait, les bovins lait-viande (avec presque 40\% des exploitations) loin devant l'orientation polyélevage granivores ou l'orientation grandes cultures et herbivores (presque $25 \%$ des exploitations). Les orientations bovins viande et autres herbivores utilisent donc des équipes conjugales où la conjointe est peu mise à contribution (moins qu'à mi-temps) et qui ne sont donc pas très éloignées des équipes qui se réduisent au seul chef d'exploitation.

Les équipes conjugales sont surreprésentées dans les exploitations de taille économique moyenne $(43,1 \%$ d'équipes conjugales dans les exploitations de 60 à 150 hectares-équivalent-blé). Leur importance se réduit dans les plus petites et les plus grandes exploitations. Elles ne représentent plus que 21,7\% des équipes dans les exploitations de

\footnotetext{
7 Rappelons que cette typologie est fondée sur la participation productive du conjoint et la présence de salariés. Elle ne prend pas en compte l'éventuelle présence d'un fils ou d'un parent retraité sur l'exploitation. Sur ce point, la participation de la main-d'œuvre familiale non coexploitante (c'est-à-dire sans statut) représente en 2010 moins de 5\% du travail total des exploitations et s'est encore réduite par rapport à 2000 (Ministère de 1'Agriculture 2011). Cela montre moins la faiblesse des aides des ascendants ou descendants que le fait que leur participation se fait aujourd'hui plus souvent dans un cadre professionnel statutaire.
} 
moins de 12 hectares-équivalent-blé et $11,3 \%$ des équipes des exploitations de plus de 375 hectares-équivalent-blé. Elles sont légèrement surreprésentées au sein des exploitations individuelles (32,5\% des exploitations individuelles ont ce type d'équipe contre $21,5 \%$ des GAEC et $25,4 \%$ des EARL).

Parmi les secteurs où les équipes non conjugales et non salariées sont les plus importantes, on retrouve sans grande surprise les céréaliculteurs et les orientations d'élevage peu intensif (comme bovins viande et poly-élevages herbivores). Les céréaliculteurs se distinguent des seconds car ils forment moins d'équipes conjugales et font plus appel au salariat (quoique dans une proportion moins importante que la moyenne). L'importance économique des premiers leur permet de recourir au salariat. En effet, le taux des équipes salariées ou des équipes conjugales et salariées croît continument en fonction de la dimension économique de l'exploitation : $68,6 \%$ des exploitations de moins de 12 hectares-équivalent-blé adoptent des équipes ni conjugales ni salariées contre $11,1 \%$ des exploitations de plus de 375 hectares-équivalent-blé. Les exploitations individuelles sont plus fréquemment que les exploitations sociétaires porteuses de ce type d'équipe sans conjointe ni salarié mais qui peut comporter d'autres membres de la famille ou des voisins $(41,8 \%$ sont dans ce cas contre $29,8 \%$ des GAEC et $15,3 \%$ des EARL). De même, les exploitations individuelles sont deux fois moins enclines que les exploitations sociétaires à développer des équipes salariées.

Une autre forme d'équipe conjugale est caractérisée par un modèle qui combine travail conjugal et travail salarié. Certains résultats vus précédemment peuvent être précisés : les secteurs de production les plus spécialisés sont aussi les plus enclins à avoir des équipes conjugales et salariées ${ }^{8}$. Mais il faut dis- tinguer les orientations maraîchage, horticulture ou vin de qualité dans lesquelles cette forme d'équipe de travail est très présente (de 35 à $45 \%$ des exploitations), des orientations fruits, autre viticulture, où elle est à peine plus présente que la moyenne.

Formée d'équipes limitées à la maind'œuvre familiale ou s'articulant à une main-d'œuvre salariée importante, l'agriculture conjugale montre ses capacités d'adaptation aux contraintes des différentes orientations productives. Cette formation sociale ne cesse cependant de reculer au profit d'une agriculture non conjugale comme en témoigne le tableau 4.

Si le mouvement de «déconjugalisation» est très net, deux choses doivent être rappelées :

- Certaines orientations sont plus particulièrement enclines à développer des équipes qui donnent une place aux conjointes.

- Si les conjointes travaillent sur les exploitations agricoles, celles qui restent sur l'exploitation ont plus tendance en 2000 qu'en 1988 à travailler à temps plein. Elles disposent de surcroît de statuts professionnels de plus en plus reconnus grâce à l'essor du statut d'associé rendu accessible avec l'instauration de l'EARL en 1985. Rattin (2002b) propose le terme de «reconjugalisation ${ }^{9} \gg$ pour désigner ce renforcement du travail de la conjointe dans une frange importante des équipes conjugales.

Ces deux constatations nous conduisent à penser que le secteur de l'élevage bovin laitier ou bovin laitier et à viande reste un milieu professionnel où les équipes conjugales sont les plus fréquentes, où l'engagement de la conjointe est très important (plus souvent de l'ordre d'un mi-temps que dans les autres secteurs) et où les équipes de travail font très peu appel au salariat (même si les services de remplacement et les groupements d'employeurs se sont développés dans certaines régions). Dans ces équipes de travail les moins touchées par la «déconjugalisation» et qui pourraient connaître certaines formes de «reconjugalisation», comment sont perçues aujourd'hui les conditions de travail des femmes et cette situation de collaboration en famille ?

\section{2 / La place des femmes dans les élevages laitiers : différenciation selon les trajectoires sociales et bio- graphiques}

Nous analysons la répartition des tâches et des responsabilités, significative de la structure familiale du travail (Barthez 1982), et les représentations que les femmes ont de leur travail afin de mettre au jour leur rôle et leur place dans les élevages laitiers, secteur où les équipes conjugales sont les plus fréquentes comme l'a montré l'analyse statistique. Le travail d'enquête auprès des femmes a été mené dans le Ségala, région naturelle dans laquelle l'élevage bovin laitier est très présent, avec une forte dimension familiale. En effet, dans la petite région du Ségala (Nord, NordOuest du département de l'Aveyron et Est du département du Lot), l'élevage bovin laitier s'est développé dans les années 1960, 1970 avec la volonté des responsables professionnels agricoles, issus de la Jeunesse agricole catholique de maintenir des exploitations de taille moyenne, modernisées, à dimension familiale. Aujourd'hui, si ce modèle n'est plus la référence unique (Cournut et al 2008), les exploitations restent des entreprises familiales avec une forte proportion d'exploitations individuelles dans le Lot $(78 \%)$ (Agreste $2011 \mathrm{a})$ et une augmentation des formes sociétai-

Tableau 4. Evolution de la répartition des types d'équipe de travail entre 1988 et 2000 (en \%), pour les exploitations dont le chef d'exploitation ou le premier coexploitant est un homme (Source : RGA 1988 et RA 2000).

\begin{tabular}{|l|c|c|c|c|}
\hline & Equipe conjugale & $\begin{array}{c}\text { Equipe conjugale } \\
\text { et salariée }\end{array}$ & $\begin{array}{c}\text { Equipe salariée } \\
\text { non conjugale }\end{array}$ & $\begin{array}{c}\text { Equipe non conjugale } \\
\text { non salariée }\end{array}$ \\
\hline 1988 & 42,7 & 26,5 & 10,9 & 19,9 \\
\hline 2000 & 30,3 & 18,1 & 14,1 & 37,5 \\
\hline
\end{tabular}

\footnotetext{
${ }^{8}$ Le lien déjà constaté plus haut entre le salariat et la dimension économique est toujours valide : $3,1 \%$ des équipes sont conjugales et salariées dans les exploitations de moins de 12 hectares-équivalents-blé contre 43,1\% dans les exploitations de plus de 375 hectares-équivalent-blé. On retrouve également le lien entre statut juridique de l'exploitation et la configuration des équipes : $14,8 \%$ des exploitations individuelles ont une équipe conjugale et salariée contre $21,1 \%$ des GAEC et $36,5 \%$ des EARL.

${ }^{9}$ Ce terme de «reconjugalisation» est ambigu car il insiste surtout sur le statut conjugal de la travailleuse. Or, subjectivement, celle-ci n'a pas forcément l'impression qu'elle travaille à titre de «conjointe» sur l'exploitation. La deuxième partie analysera plus avant les perceptions des femmes dans cette situation.
} 
res en Aveyron, notamment dans les élevages bovins laitiers (Agreste 2011b). Les exploitations de ces départements, 57 ha en Aveyron, 44 ha dans le Lot, sont de taille moyenne pour la région Midi-Pyrénées (48 ha, Agreste 2011c), les exploitations enquêtées, avec 63 ha en moyenne ayant toutefois une taille légèrement plus importante que la moyenne de chacun des deux départements. Dans cette période de forte restructuration de l'élevage laitier, la dynamique qu'a eue cette région durant les différentes phases de modernisation de l'agriculture lui confère un intérêt spécifique. Bien qu'ayant des capacités d'investissement modérées, la modernisation des élevages se poursuit mais le recours à l'automatisation, notamment avec les robots de traite, reste encore limité.

Le travail des femmes à l'extérieur de l'exploitation se développe (1/4 des couples sont concernés) (Cournut et al 2008), mais en proportion moindre qu'au niveau national (64\% des femmes de moins de 40 ans travaillent à l'extérieur) (Agreste GraphAgri 2003). Dans ces départements, à fort caractère rural, les opportunités d'emploi pour les femmes se concentrent sur certains secteurs. Près d'une femme sur deux (47\% dans le Lot, $48 \%$ en Aveyron) est «employée» et une sur cinq exerce une «profession intermédiaire» (assistante sociale, institutrice, infirmière) (Mura 2002a et b).

Dans douze des quatorze exploitations enquêtées, les équipes de travail se limitent à la famille et surtout au couple d'exploitants et font appel au salariat pour moins de $20 \%$ des UTA. Elles illustrent la configuration conjugale dans laquelle les conjointes travaillent plus qu'à mi-temps. Dans les deux autres exploitations, les équipes de travail mêlent travail familial, conjugal et salarié, l'une en raison de l'engagement professionnel du chef d'exploitation, l'autre en remplacement de l'épouse qui a décidé de retourner travailler à l'extérieur. Dans ce contexte, quelles représentations les femmes ont-elles de leur travail ? Quelle est leur place et leur rôle ?

\section{1 / Participation des femmes aux équipes conjugales de tra- vail}

Trois modes de participation féminine aux équipes conjugales de travail peuvent être différenciés selon que les femmes s'installent sur l'exploitation de leur mari au moment du mariage, après une expérience professionnelle ou qu'elles s'installent sur l'exploitation de leurs parents.
Le premier mode de participation correspond à l'installation sur l'exploitation du mari, au moment du mariage ou peu de temps après (profil 1: 7 femmes/14). L'installation sur la ferme du conjoint au moment du mariage semble aller de soi. Les femmes de ce profil ont effectué des études courtes ou techniques (5/7 sont titulaires d'un baccalauréat général ou agricole) et n'ont pas d'expérience professionnelle en dehors de l'agriculture au moment du mariage. Leur famille leur a transmis un capital culturel qui les a familiarisées avec la culture agricole (6/7 ont des parents agriculteurs) et leur souhait est de travailler sur une exploitation. Deux femmes ont une expérience professionnelle (ouvrière, caissière) assez courte (entre 2 et 3 ans) à la suite de leurs études. Des facteurs concomitants : la situation économique des exploitations plutôt favorable (6/7 ont entre 300000 litres et 400000 litres de quotas laitiers), le besoin de main-d'œuvre pour le troupeau, le manque d'emplois locaux, mais également le souhait de conjuguer vie familiale et vie professionnelle avec une forte disponibilité pour les enfants et pour la famille, font que ces femmes adoptent le projet professionnel de leur mari, sans trop se poser de questions.

«Je suis une fille d'agriculteur, j'ai fait une école d'agriculture pour m'installer sur la ferme de mes parents et entre temps, $j$ 'ai rencontré mon mari et on s'est installé tous les deux sur son exploitation, voilà... Je ne me suis même pas posée la question d'aller faire autre chose. Voilà, ça m'a toujours plu» (Nadia, coexploitante, EARL, 42 ans, Baccalauréat Technique Agricole, beaux-parents sur l'exploitation).

Dans la deuxième forme de participation (profil $2: 5 / 14$ ), les femmes s'installent à la suite d'une expérience professionnelle significative (3/14) : technicienne au contrôle laitier, employée de bureau à la MSA, animatrice syndicale, ou directement à la suite de leur formation plus longue que celle du profil 1 (2/14). Leur décision de s'installer peut être liée à des évènements particuliers dans la trajectoire de l'exploitation ou/et à la difficulté à trouver un emploi localement en lien avec leur qualification.

«Je n'étais pas issue du milieu agricole... J'ai fait un BAC et un BTS, et je cherchais du boulot dans cette branche, je n'en ai pas trouvé, j'ai été caissière dans un supermarché deux ans, et un jour, on a eu une opportunité de reprendre l'exploitation de nos voisins, donc mon mari m'a proposé de m'installer avec lui. (...) Il a fallu que je fasse une formation adulte, après $j$ 'ai fait un stage de 6 mois et après je me suis installée dans le GAEC avec mon mari et ma belle-mère» (Irène, parents non agriculteurs, coexploitante GAEC, 35 ans, BTS informatique, beaux-parents sur l'exploitation).

Marie (parents agriculteurs, coexploitante EARL, 47 ans, BAC) a quitté son emploi à la MSA pour s'occuper de ses jeunes enfants, puis suite à la dissolution de la société avec les beaux-frères, elle décide de s'installer pour soulager son mari : «il se retrouvait tout seul avec une charge de travail, c'est pour ça que j'ai pris la décision».

Ces femmes ne sont pas issues du milieu agricole (4/5). Elles disposent d'un capital scolaire plus élevé que celles du profil 1 (4/5 sont titulaires d'un BTS). Elles souhaitent, comme les précédentes, consacrer du temps à la vie familiale.

Un troisième mode de participation correspond aux femmes qui s'installent sur l'exploitation de leurs parents (profil $3: 2 / 14)$. Ces installations se font souvent après une première expérience professionnelle et correspondent à un choix personnel. Le fait de ne pas trouver de travail dans sa branche a décidé Véronique (40 ans) à s'installer sur 1'exploitation familiale qui, par ailleurs, n'a pas de repreneur.

"J'ai un BAC informatique, je n'ai pas trouvé de travail dans ma branche, j'ai fait des petits boulots par ici, notamment du travail en usine, ce qui m'a permis de réaliser que ce n'était pas comme ça que je voyais mon avenir... Donc j'ai galéré pendant une dizaine d'années et puis après, puisque personne restait sur l'exploitation, je suis repartie passer mes diplômes agricoles et voilà».

L'installation est motivée par la valeur patrimoniale et sentimentale de l'exploitation, fortement exprimée, contrairement aux profils précédents : "Ils ont été très fiers que je puisse m'installer sur le domaine familial... c'est quelque chose de profond pour eux d'avoir transmis un outil de travail et un domaine qui a été viable jusqu'à aujourd 'hui» (Céline, chef d'exploitation individuelle, 42 ans, BAC).

Pour chacun des profils, nous analysons la répartition des tâches et des responsabilités et les représentations que les femmes ont de leur travail afin de caractériser l'engagement familial dans les exploitations.

\section{2 / Répartition des tâches et des responsabilités}

Dans les exploitations du profil 1 , la répartition du travail entre mari et 
femme correspond dans les grandes lignes à une division sexuelle classique du travail: les hommes effectuent une partie du travail d'élevage, les cultures et prennent les décisions stratégiques de l'exploitation tandis que les femmes participent à la traite, donnent les soins aux veaux et font le travail administratif. Elles travaillent quotidiennement dans les élevages en effectuant une grande partie du travail d'astreinte, mais sans être entièrement responsables dans la mesure où leur mari y prend part également et gère l'ensemble des activités. Leur travail n'a alors pas ou peu de visibilité. Les femmes de ce profil peinent à «trouver leur place», comme elles l'expliquent, c'est-à-dire qu'elles n'ont pas d'espace de travail à elles sur l'exploitation. Elles assument en grande partie le travail domestique et l'éducation des enfants.

Muriel (conjointe-collaboratrice, 46 ans, parents agriculteurs, BAC, pas d'expérience professionnelle, un de ses fils s'installe) a toujours travaillé sur 1'exploitation familiale. Sans autre expérience professionnelle, l'engagement de Muriel sur l'exploitation est d'abord conjugal : "Je suis sur l'exploitation parce que j'ai épousé un agriculteur, je l'ai suivi sur l'exploitation...». Fille d'agriculteur, elle a pourtant eu des difficultés à savoir comment participer à la vie de l'exploitation : "J'aurais pu peut-être ou peut-être pas m'investir dans les champs à une époque, davantage... je ne me sentais pas à ma place, je ne trouvais pas ma place».

«Trouver sa place», c'est rechercher un espace de travail dont on serait responsable, un ensemble d'activités, un espace à soi. Muriel en semble dépourvue : "Je participe à la traite matin et soir, c'est un coup de main, on y va à deux (avec mon mari), je ne suis pas indispensable».

Aide de son mari sur plusieurs espaces, elle n'en maîtrise ou contrôle vraiment aucun. Sa part de travail qui ne lui appartient plus devient alors invisible, ce qu'elle constate amèrement : «Je pense que malheureusement, une femme sur l'exploitation, on n'est pas assez reconnue, surtout quand on est conjointe».

Muriel apprécie cependant positivement sa situation pour la qualité de vie familiale "L'avantage d'une femme sur une exploitation, c'est de pouvoir élever ses enfants. Même si on est pris par le boulot, on est présent pour les enfants... au niveau du couple, on est tout le temps ensemble. On a même un privilège, parce qu'à midi, on prend le repas ensemble».

Les femmes de ce profil ont conscience de faire partie d'une minorité : celles de femmes qui travaillent avec leur mari agriculteur. En s'exprimant sur le travail extérieur d'autres femmes, ces femmes évoquent les questions de reconnaissance et d'autonomie financière.

"Aujourd'hui, on trouve beaucoup de femmes qui n'hésitent pas à épouser un agriculteur, mais à condition de travailler à l'extérieur. Il y en a très peu qui veulent venir sur l'exploitation, parce qu'on n'est pas assez valorisée. C'est un manque de reconnaissance aussi (...) On est dépendante du mari, on n'a pas une feuille de paie à la fin du mois, on n'a pas d'indépendance» (Muriel).

Les femmes, installées à la suite d'une expérience professionnelle significative et/ou avec un capital scolaire élevé (profil 2), effectuent également la traite, les soins aux veaux et le travail administratif, mais à la différence des femmes du profil 1, elles se battent plus fortement pour acquérir une autonomie et une reconnaissance professionnelle. Elles assument une grande partie du travail domestique, mais dans un rapport avec le conjoint qui est discuté. Un effort de clarification des responsabilités de chacune et de chacun sur l'exploitation a été accompli. Ces femmes cherchent à optimiser l'organisation du travail pour du temps libre et des congés. Afin de répondre à leurs aspirations à un travail défini et reconnu, elles prennent la responsabilité d'une partie de la conduite du troupeau ou bien développent une activité propre.

Ainsi, Irène (coexploitante GAEC, 35 ans, non issue du milieu agricole), titulaire d'un BTS en informatique, a appris le métier lors de la formation à l'installation et avec ses beaux-parents. Aujourd'hui, elle assume pleinement cette tâche : "la traite, puisque c'est ça que je fais à $100 \%$, que je maîtrise le mieux donc... c'est vraiment mon travail quoi, je connais mes vaches par cœur».

Marie (coexploitante EARL, 47 ans, $\mathrm{BAC}$, parents agriculteurs), installée à la suite d'une expérience professionnelle qu'elle a fortement appréciée, ressent, après quelques années sur l'exploitation, une perte d'autonomie par rapport à sa situation antérieure. Pour «se démarquer de son mari, ne plus être dans l'ombre» Marie développe alors un élevage de lapins "c'est quelque chose qui m'appartient, qui me revalorise un peu... mais ça m'empêche pas de traire tous les soirs en principe». Dès lors, elle participe aux groupes professionnels, prend des responsabilités et se sent reconnue «Je suis au Conseil d'administration $d u$ groupement des éleveurs de lapins, je me rends compte qu'on a besoin de s'investir pour faire avancer les choses et rencontrer des gens du métier» (Marie, coexploitante EARL, 47 ans). D'une certaine manière Marie a construit sa place en marge de l'exploitation avec cet atelier lapin 10 quand Irène l'a construite sur l'exploitation avec le contrôle total de «l'atelier de traite».

Cependant, l'autonomie professionnelle sur l'exploitation n'est pas toujours facile à acquérir. L'histoire de Lucie est révélatrice des difficultés que rencontrent certaines femmes pour se faire une place au sein d'une équipe où se mêle travail conjugal et familial. Lucie (coexploitante GAEC, 45 ans, parents agriculteurs, BTS horticulture) s'installe à la suite de plusieurs expériences professionnelles. Lorsqu'elle quitte son emploi pour travailler dans l'exploitation familiale, elle choisit de développer sa propre activité. Elle monte un projet de fromagerie, mais celui-ci ne marche pas en raison des exigences trop lourdes de la grande distribution. L'échec de son projet, et les relations difficiles avec la belle-famille, font que Lucie décide de quitter l'exploitation pour monter sa propre entreprise de décoration florale et être à nouveau autonome et indépendante.

Les femmes de ce profil ont essayé et sont parvenues (au moins pendant un temps) à se construire une place dans un contexte de travail conjugal. Leur capital scolaire et culturel leur confère les ressources nécessaires à cette construction. Mais les places construites sont toujours fragiles et si elles sont remises en question comme dans l'exemple de Lucie, les femmes de ce profil n'hésitent pas à utiliser ces capitaux pour acquérir une activité à elles et une reconnaissance professionnelle hors de l'exploitation.

Les femmes, qui ont repris l'exploitation de leurs parents (profil 3), font le projet de s'installer avec la volonté d'être indépendantes professionnellement. Elles assurent la conduite du troupeau et

\footnotetext{
${ }^{10}$ Les activités agri-touristiques comme les chambres d'hôtes peuvent aussi être un de ces ateliers en marge de l'activité agricole où les femmes trouvent une forme d'autonomie professionnelle (Giraud 2001). Les initiatives des femmes d'agriculteurs aveyronnaises en matière de tourisme vert, de transformation de produits agricoles ou de vente directe, sont exposées dans la durée par Marie-Thérèse Lacombe (2009).
} 
effectuent tout ou partie du travail du sol et de la conduite des cultures. Toutes deux cherchent à diminuer les contraintes de travail en optant pour la traite une fois par jour durant certaines périodes ou en déléguant une partie des travaux des cultures, afin de disposer de temps pour autre chose. A la différence des profils 1 et 2 , ces femmes gèrent la totalité de la sphère productive de l'exploitation et prennent les décisions stratégiques. Elles se caractérisent par leur capacité à se détacher des situations de dépendance vis-à-vis du conjoint et de la famille (Rieu et Dahache 2008) comme l'illustre l'histoire de Véronique (chef exploitation EARL, 40 ans, BAC informatique). Véronique et Paul ont chacun repris l'exploitation de leurs parents, l'une avec un élevage laitier, l'autre avec un élevage à viande, mais ils travaillent séparément. Cette configuration sera amenée à évoluer en fonction de la rentabilité des deux élevages, mais également pour répondre aux aspirations du couple de travailler ensemble : "on n'est jamais ensemble, ce n'était pas tout à fait ce qu'on avait prévu, puisque l'on est chacun sur nos exploitations espacées de $6 \mathrm{~km}$... enfin, je veux dire, on ne travaille pas vraiment ensemble».

Véronique, tout comme Céline, font partie de groupes professionnels au sein desquels elles ont pris des responsabilités. De leurs points de vue, il est toujours difficile pour une femme d'être reconnue professionnellement dans le secteur agricole. Les diverses instances professionnelles sont largement dirigées par les hommes, et les représentations masculines sur le rôle des femmes sont toujours présentes : "le milieu d'hommes, il est assez fermé, la femme (rire), c'est plutôt la femme, elle fait à manger, elle s'occupe des enfants, par là quelques génisses, et puis c'est tout». Mais, elles sont parvenues à négocier de nouveaux rapports entre les hommes et les femmes (Rieu et Dahache 2008).

\section{3 / Discussion - Conclusion}

Nous avons tenté d'interroger le travail conjugal dans les exploitations. Les résultats de l'analyse statistique mettent en évidence des réalités contrastées selon les secteurs de production, la dimension économique des exploitations et les caractéristiques des équipes de travail. Quatre grands secteurs se dessinent. Une agriculture qui est centrée sur le travail conjugal et fait peu appel au salariat, particulièrement présente dans les exploitations d'élevage, surtout en bovins laitiers ou laitiers et à viande. Dans ces orientations, le travail d'astreinte est important et la division sexuelle du travail marquée. Une agriculture qui s'appuie peu sur le travail des conjointes et peu sur le salariat, et qui est fortement représentée dans les exploitations céréalières et les élevages d'autres herbivores. Les conjointes ont alors plus souvent qu'ailleurs une carrière professionnelle en dehors des exploitations. Une agriculture où les quatre types d'équipe se rencontrent dans des proportions similaires, y compris le recours au salariat seul qui est donc un peu plus fréquent que dans les deux précédents secteurs. Ce groupe rassemble les orientations de cultures générales, de granivores, de viticulture, ou de fruit. Enfin, une agriculture spécialisée (comme les productions maraîchères, horticoles ou la viticulture de qualité) où les équipes sans conjoint et sans salarié sont très peu fréquentes. Les conjointes peuvent faire équipe avec le chef d'exploitation (pour le maraîchage et l'horticulture) mais travaillent surtout plus souvent qu'ailleurs aux côtés de salariés nombreux. Le secteur laitier représente une situation emblématique du travail conjugal.

De nombreuses recherches ont étudié la place des femmes dans les exploitations familiales et ont montré la diversité des situations (Brandth 2002). Les enquêtes dans les exploitations du Ségala ont permis de mieux cerner la position sociale et la place des femmes dans les équipes de travail conjugales. Trois profils ont été identifiés. Dans le profil 1, les femmes se sont installées sur l'exploitation de leur mari au moment du mariage. Elles effectuent de multiples tâches pour la famille et pour l'exploitation. A la différence de leurs mères, elles ont un statut, mais la répartition du travail entre mari et femme n'est pas discutée, elles subissent les stéréotypes de sexe et leur travail agricole est peu valorisé. Ces femmes sont dans une situation d'aides familiales disposant de peu d'autonomie. Cette situation peut être analysée dans le cadre de la théorie des ressources (Blood et Wolfe 1960, Glaude et Singly 1986) qui explique le partage des tâches au sein des couples en fonction de la détention par chaque conjoint de certains capitaux. Les femmes qui sont dépourvues de capitaux culturels, et n'ont pas d'expérience longue et valorisante de salariat sont plus enclines à se retrouver dans ce profil car elles disposent de peu d'armes pour construire leur place sur l'exploitation. Leur place dans les exploitations agricoles est peu visible et rappelle la condition de la plupart des femmes en agriculture jusque dans les années 1980 où, quelle que soit leur participation aux travaux agricoles, elles étaient considérées comme des aides familiales (Barthez 1982).

Les profils 2 et 3 montrent des formes possibles de professionnalisation pour les femmes qui optent pour le travail dans les élevages laitiers.

Les femmes du profil 2, installées sur l'exploitation de leur mari, prennent la responsabilité d'une partie de la conduite $\mathrm{du}$ troupeau ou développent une autre activité sur l'exploitation. Leur expérience professionnelle valorisante ainsi qu'une origine sociale et un capital scolaire et culturel plus élevés que les précédentes, leur confèrent les ressources nécessaires pour construire leur place dans le pôle de la production de l'exploitation ou en marge de l'exploitation. Elles développent leur projet professionnel pour être autonomes et reconnues professionnellement. Ces femmes s'affirment comme «sujet» de leurs actions et ressentent positivement la place et le statut qu'elles occupent. Certaines obtiennent une reconnaissance de leurs compétences et un statut dans une activité centrale pour l'exploitation (la production laitière avec la maitrise de la traite) tandis que d'autres développent une nouvelle activité en marge de celle-ci (Giraud et Rémy 2013).

Le troisième profil caractérise les femmes qui s'installent sur l'exploitation de leurs parents. Elles exercent leur métier dans toutes ses dimensions, développent leur projet professionnel et obtiennent une reconnaissance professionnelle. Ces femmes se situent en décalage par rapport aux déterminants de genre des environnements socialisateurs agricoles. La manière dont elles exercent le métier, notamment avec une gestion optimisée du temps de travail, ainsi que leurs responsabilités dans les organisations professionnelles, contribuent à faire évoluer les modèles professionnels familiaux et conjugaux (Rieu et Dahache 2008).

Nous mettons en évidence les inégalités des situations féminines dans le contexte du travail conjugal. Il ressort que les conditions d'émancipation des femmes sont liées à la formation et à l'expérience professionnelle en dehors de l'exploitation. Dans cette configuration de travail conjugal avec des stéréotypes de sexe très présents, les femmes qui disposent d'un capital culturel plus élevé parviennent à construire des espaces de travail où elles peuvent être reconnues personnellement et professionnellement 11 . Leurs stratégies répondent à leurs besoins individuels d'autonomie et

11 Cette situation d'autonomie dans la dépendance est loin d'être unique comme le montre le cas très proche des femmes de préfets étudié par Singly et Chaland (2002). 
au développement des exploitations familiales (Schmitt et Inhetveen 2010).

Ce travail montre des formes différenciées de professionnalisation pour les femmes dans un des secteurs agricoles les moins touchés par la «déconjugalisation». Dans un contexte où les dimensions familiales et professionnelles restent fortement imbriquées, des femmes parviennent à faire reconnaître leurs compétences en puisant dans leur histoire singulière. Notre analyse statistique et notre typologie restent cependant très globales. D'autres auteurs ont bien montré combien l'engagement des femmes sur l'exploitation devait être mis en relation avec le cycle de vie des femmes, mais aussi avec les nécessités de la transmission des exploitations. Certaines femmes peuvent «faire la soudure» entre la retraite du mari et la reprise par un successeur (Cardon 2004), une situation que nous avions exclue de l'analyse statistique et qui devrait enrichir la typologie des équipes conjugales ou de la participation des femmes au travail de l'exploitation.
L'analyse statistique devrait prendre également en compte la participation parentale au travail de l'exploitation qui, comme on l'a vu dans l'enquête ethnographique, a un rôle souvent négatif pour les conjointes qui veulent s'investir sur l'exploitation, mais aussi d'autres interactions comme l'aide domestique des beaux-parents qui rend possible le travail à l'extérieur des jeunes conjointes (Bessière 2008).

Quel avenir alors pour le travail conjugal dans les exploitations laitières en constante modernisation ? La révolution technologique et l'usage de l'informatique en élevage permettent une meilleure gestion des ressources, un suivi précis des performances de chaque animal et l'automatisation croissante des tâches, notamment de la traite, avec la diffusion du robot. On a pu dire, avec quelque véracité, que la multiplication des salles de traite chassait les femmes de l'étable. Certaines cependant ne se sont jamais résolues à renoncer à ce métier. Celles qui n'avaient pas d'autres choix social que de rester femmes d'agriculteur, celles qui ont pu, ont su, uti- liser leurs capitaux pour construire une place à soi sur ou en marge de l'exploitation agricole. Ces femmes qui cherchent à défendre leur autonomie professionnelle ne sont pas si éloignées de celles qui ont recherché une autonomie professionnelle en quittant l'exploitation agricole car au cœur de leur démarche commune il y a la recherche de la défense et de la reconnaissance de l'individu.

\section{Remerciements}

Nous remercions chaleureusement Jacques Rémy, sociologue, directeur de recherche à l'INRA, pour son soutien et ses conseils avisés tout au long de l'écriture de cet article. Nous remercions également Virginia Courdin, de la Faculté d'agronomie de Paysandù (Uruguay), pour son excellent travail d'enquête réalisé au cours d'un stage de Master 2, ainsi que nos deux relecteurs anonymes dont les remarques ont grandement aidé à améliorer la première version de ce texte.

\section{Références}

Agreste, 2003. GraphAgri, 161p.

Agreste, 2011a. Recensement agricole 2010, Données Lot, 62, 4p.

Agreste, 2011b. Recensement agricole 2010, Données Aveyron, 59, 4p.

Agreste, 2011c. Recensement agricole 2010, Midi-Pyrénées, 4p.

Barthez A., 1982. Famille, Travail et Agriculture. Economica, Paris, France, 189p.

Bessière C., 2008. «Travailler à l'extérieur» des implications ambivalentes pour les compagnes d'agriculteurs. Nouvelles questions féministes, 27, 2, 53-65.

Bessière C., 2010. De génération en génération, Arrangements de famille dans les entreprises viticoles de Cognac. Raisons d'agir, Paris, France, 215p.

Bessière C., Giraud C., Renahy N., 2008. Famille, travail, école et agriculture. Revue d'Etudes en Agriculture et Environnement, 88, 3, 5-19.

Blood R.O., Wolfe D.M., 1960. Husbands and Wives, the dynamics of married living. The free press, New York, USA, 293p.

Brandth B., 2002. Gender identity in european family farming : A Literature Review Sociol. Rural, 42, 181-200.

Cardon P., 2004., Des femmes et des fermes. Genre, parcours biographiques et transmission familiale. Editions L'Harmattan, Paris, France, $311 \mathrm{p}$.

Cournut S., Hostiou N., Pailleux J.Y., Leger L., 2008. Quelles adaptations des systèmes laitiers pour réduire la contrainte «travail» ? Une étude en Ségala, Renc. Rech. Rum., 15, 163166.
Dedieu B., Servière G., 1999. Caractériser et évaluer l'organisation du travail en élevage Fasade, 1, 4p.

Desriers M. 2007. L'agriculture française depuis cinquante ans : des petites exploitations familiales aux droits à paiement unique. Agreste Cah., 2, 3-14.

Giraud C., 2001. Chambres d'hôtes à la ferme et autonomie de la femme en agriculture. Thèse de doctorat de sociologie, Université Paris Descartes.

Giraud C., Rémy J., 2013. Division conjugale du travail et légitimité professionnelle. Le cas des activités de diversification agricole en France. Travail genre et société, 30 , à paraître.

Giroux G., 2011. Recensement agricole 2010, Premières tendances. Agreste Recensements agricoles, $266,4 \mathrm{p}$.

Glaude M., Singly F., 1986. L'organisation domestique : pouvoir et négociation. Econ. Stat., 187, 3-30.

Harff Y., Lamarche H., 1998. Le travail en agriculture : nouvelles demandes, nouveaux enjeux. Econ. Rurale, 244, 3-11.

Hervieu B., Purseigle F., 2009. Pour une sociologie des mondes agricoles dans la globalisation. Etudes rurales, 183, 177-200.

Lacombe M.T., 2009. Pionnières ! Les femmes dans la modernisation des campagnes de l'Aveyron de 1945 à nos jours. 192p.

Muller P,. 2009. Le basculement du regard. Etudes rurales, 183, 101-112.

Mundler P., Laurent C., 2003. Flexibilité du travail en agriculture : méthodes d'observation et évolutions en cours. Ruralia, 12/13, 239-257.
Mura B., 2002a. Regards sur le Lot. INSEE Midi-Pyrénées, 9-46, 2p.

Mura B., 2002b. Regards sur l'Aveyron. INSEE Midi-Pyrénées, 9-12, 2p.

Rattin S., 2002a. L'agriculture au féminin se professionnalise. Agreste Cah., 2, 15-22.

Rattin S., 2002b. Les ménages d'agriculteur en 2000 : un sur trois est pluriactif. Agreste Cah., 2, 3-14.

Rattin S., 2006. Actifs familiaux professionnels : les deux tiers sont des exploitants. Agreste Cah., 4, 13-22.

Rieu A., Dahache S., 2008. S'installer comme agricultrice : sur la socialisation et la formation sexuée en agriculture. Revue d'Etudes en Agriculture et Environnement, 88, 71-94.

Rémy J., 2010. Un métier en transformation. Introduction. In : Les mondes agricoles en politique. De la fin des paysans au retour de la question agricole. Hervieu B., Mayer N., Muller P., Purseigle F., Rémy J. (Eds). Les Presses de Sciences Po, Paris, France, 41-50.

Schmitt M., Inhetveen H., 2010. Balancing uncertainties - Women farmers on small-and medium scale enterprises face to modernization of agriculture. $9^{\text {th }}$ Eur. IFSA Symp., 11291137.

Singly de F., 2005. Le soi, le couple et la famille. Editions Colin, Paris, France, 255p.

Singly de F., 2007. L'individualisme est un humanisme. Editions de l'Aube, La Tour d'Aigues, 127p.

Singly de F, Chaland K., 2002. Avoir le «second rôle» dans une équipe conjugale. Revue Française de Sociologie. 43, 127-157. 


\title{
Résumé
}

Cet article contribue au débat sur la dimension conjugale du travail en agriculture. L'analyse repose sur deux approches complémentaires : une analyse des données du recensement agricole et une enquête qualitative auprès de femmes qui travaillent dans les élevages laitiers du Ségala. La première partie dresse un panorama global des types d'équipes de travail et de leurs évolutions selon les productions. Elle caractérise les différents modes d'organisation selon leur caractère plus ou moins conjugal. Il ressort que le secteur de l'élevage bovin laitier ou bovin à viande reste un milieu professionnel où les équipes conjugales sont les plus fréquentes et où l'engagement de la conjointe est très important. A partir des enquêtes, l'analyse de la place et du rôle des femmes montre trois modalités de participation selon que les femmes s'installent sur l'exploitation du mari au moment du mariage ou après une expérience professionnelle, ou qu'elles s'installent sur l'exploitation de leurs parents. Dans la première modalité les femmes n'ont pas d'espace de travail à elles sur l'exploitation, dans la deuxième, les femmes sont autonomes et responsables d'une activité, et dans la troisième les femmes sont gestionnaires de toute la sphère productive de l'exploitation. Les femmes dotées d'une formation scolaire élevée et d'une expérience professionnelle valorisante en dehors de l'exploitation, sont en mesure de questionner la division sexuelle du travail et de construire leur place dans ces configurations conjugales de travail. Cela nous amène à nous interroger sur la place nouvelle des individus, notamment des femmes, dans les exploitations agricoles.

\begin{abstract}
Is work on dairy farms still a family affair?

In this article the aspect of couples working together in agriculture was studied. Two complementary approaches were used in the analysis: one based on agricultural census data and one qualitative study of women working on dairy farms in the Segala region (south- west of France). In the first part, a general overview is given of the different types of work groups and changes undergone according to their production orientations. The first characteristic to be taken into account was if the farm is run by a couple (the socalled family farm) or by another kind of organization or partnership. The sector of dairy farming and cattle breeding remains a professional circle where couples are the most frequent working units and where the wife's commitment is especially important. According to surveys, three types of participation within the farm have become apparent: wives who work with their husbands upon marriage, or after gaining experience elsewhere or on their parents' farms. In the first type of participation, there is no clearly defined role of the wive's work on the farm, in the second one, wives work independently by managing farm activity and in the third type wives take full charge of production on the farm. Women with a higher level of education and who have work experience outside of the farm are able to question the division of tasks according to gender and to construct their own work roles within the man-wife partnership. This leads us to consider the new role of individuals, women notably, on farm units.
\end{abstract}

DUFOUR A., GIRAUD G., 2012. Le travail dans les exploitations d'élevage bovin laitier est-il toujours conjugal ? In : Numéro spécial, travail en élevage. Hostiou N., Dedieu B., Baumont R. (Eds). INRA Prod. Anim., 25, 169-180. 